\title{
Efficacy of dapoxetine treatment in Chinese patients with premature ejaculation and possible factors affecting efficacy in the real-world practice
}

\author{
Jing Peng ${ }^{1 \dagger}$, Dong Fang ${ }^{1 \dagger}$, Huixi $\mathrm{Li}^{1+}$, Yuan Tang ${ }^{1}$, Yiming Yuan ${ }^{1}$, Wanshou Cui ${ }^{1}$, Bing Gao ${ }^{1}$, Hongjun $\mathrm{Li}^{2^{*}}$ and \\ Zhichao Zhang ${ }^{1 *}$
}

\begin{abstract}
Background: The treatment effect of dapoxetine in real-world practice is not well established. This study was to investigate the factors influencing efficacy of dapoxetine for the treatment of Premature ejaculation (PE) in the realworld setting.

Methods: Altogether 154 patients were followed up between Jan 2015 and Dec 2015. The clinical global impression of change (CGIC), premature ejaculation profile (PEP), the estimated intravaginal ejaculation latency time (elELT) and estimated number of intravaginal thrusts before ejaculation (NITBE) were collected. The clinical characteristics of patients with $C G I C=0$ and $C G I C \geq 1$ were compared.

Results: After 4 weeks treatment, an obvious improvement compared with the baseline was found regarding mean eIELT $(2.4 \pm 1.6 \mathrm{~min}$ vs $1.0 \pm 0.7 \mathrm{~min}, P<0.001)$ and mean NITBE $(85.9 \pm 61.9$ times vs $37.4 \pm 28.6$ times, $P<0.001)$. The proportion of patients with a self-evaluation of at least "slightly better" and were categorized into " $\mathrm{CGIC} \geq 1$ " group was 70.1\%. There were significant differences between patients in the "CGIC $=0$ " and "CGIC $\geq 1$ " groups regarding mean NITBE $(P=0.010)$ and PEDT $(P=0.009)$ score at baseline. The adverse effects were acceptable.

Conclusion: Dapoxetine was well-tolerated and improved the sexual satisfaction of patients with PE. The severity of PE based on PEDT and NITBE suggest that there could be an effectiveness change with dapoxetine use in realworld practice.
\end{abstract}

Keywords: Premature ejaculation, Dapoxetine, Sexual dysfunction, Efficacy

\section{Background}

Premature ejaculation (PE) is one of the most of prevalent male sexual disorders, with a reported incidence of $21-33 \%$ in some populations $[1,2]$. Dapoxetine hydrochloride, a short-acting selective serotonin reuptake inhibitors (SSRIs), is the currently the only on-label oral treatment [3]. This on-demand agent has been proved to increase the quality

\footnotetext{
*Correspondence: lihongjun@pumch.cn; zhangzhichao@bjmu.edu.cn ${ }^{\dagger}$ Jing Peng, Dong Fang and Huixi Li contributed equally to this work. 2Department of Urology, Peking Union Medical College Hospital, Peking Union Medical College, Chinese Academy of Medical Sciences, No. 1 Shuaifuyuan, Dongcheng District, Beijing 100730, China

${ }^{1}$ Andrology Center, Department of Urology, Peking University First Hospital, Institute of Urology, Peking University, No. 59A, Di'anmen West St., Xicheng District, Beijing 100034, China
}

of life for the patient and their sexual partner by a pooled analysis of five randomized, placebo-controlled, phase 3 clinical trials $(N=6081)$ [4] and by a critical review [5].

Most data on the efficacy of dapoxetine are derived from clinical trials involving Western participants, and results from clinical trials may not be consistent with the results observed in the real-world practice. Clinical trials follow strict protocols involving patients with predefined inclusion criteria and results are obtained under ideal conditions. In contrast, clinical practice has several associated challenges including low rates of compliance, economic considerations, and a lack of awareness of the condition by the spouse, and these factors may influence the real-world utility of treatments for PE. In real-world 
setting dapoxetine treatment discontinuation was high. Mondaini et al. [6] reported that $68.7 \%$ of patients would discontinue dapoxetine treatment in a short time. The main reason was effect below expectations. It is important to achieve a reasonable effect in a short term.

Furthermore, stopwatch-determined intravaginal ejaculation latency time (IELT) is the most common measure to diagnose and evaluate treatment efficacy in clinical trials, which is not appropriate in real world practice. In the clinical setting, stopwatch-determined IELT is substituted by selfestimated IELT, which is often overestimated by patients [7].

In this study, we sought to evaluate the treatment effect of dapoxetine on Chinese patients with PE in the real-world practice, and to investigate factors influencing treatment efficacy of dapoxetine.

\section{Methods}

\section{Patients inclusion}

This open-label, retrospective and observational study included patients with PE who accepted dapoxetine treatment in real-world practice. All patients reported short intravaginal latency, and at least 'moderate' distress or interpersonal difficulty relating to their PE at baseline. To be included in the study, patients had to be over 18 years, and were required to be in a heterosexual, stable, and monogamous sexual relationship with the same partner for at least 6 months. The Premature Ejaculation Diagnostic Tool (PEDT) was used to diagnose PE [8] and subjects with a PEDT score $\geq 11$ were included. The linguistic validation of PEDT questionnaire has been performed and the Chinese version was used $[9,10]$. Patients were excluded from the study if they experienced primary erectile dysfunctions, engaged in sexual intercourse less than once per week, abused alcohol or illicit substances, had a history of medical or psychiatric illness, or if their partner experienced any sexual dysfunction. The study received the ethics approval by committee of Peking University First Hospital. All patients agreed and signed the informed consent, that their information (including clinical information and surveillance) would be collected for scientific study and by published in professional medical journals.

\section{Patients treatment and outcome measures}

After a 4-week run-in period, patients were asked to take dapoxetine $30 \mathrm{mg} 1-3 \mathrm{~h}$ before planned sexual intercourse. No other PE therapies were provided during the study period. Patients were assessed after 4 weeks of treatment, and were required to engage in sexual intercourse at least 6 times during the 4-week study period.

All measures including eIELT and estimated number of intravaginal thrusts before ejaculation (NITBE) were evaluated at baseline and at week 4. The definition of NITBE was the frequency of penile moving forward and backward in female vaginal. The patients were demanded using the similar frequency and depth of insertion as before and recorded the average NITBE.

Patient were required to answer the question from CGIC "Compared to the start of the study, would you describe your premature ejaculation problem as much worse, worse, slightly worse, no change, slightly better, better, or much better?" and the answers were scored from 0 to 3 (0: no change, 1: Slightly better, 2: better, 3 : much better) respectively. Patients were also assessed based on the Premature Ejaculation Profile (PEP) [11]; a validated tool that includes measures of perceived control over ejaculation, satisfaction with sexual intercourse, ejaculation-related personal distress, and ejaculationrelated interpersonal difficulty. Part of the database is attached in Additional file 1.

Patient Health Questionnaire (PHQ-9) and General Anxiety Disorder-7 (GAD-7) questionnaire were used to evaluate patients' depression and anxiety $[12,13]$. The linguistic validation of PHQ-9 and GAD-7 questionnaires have also been performed and the Chinese versions were used $[14,15]$.

\section{Statistical analysis}

Statistical analysis was carried by using SPSS 20.0 (IBM Corp, Armonk, NY, USA). Continuous variables normally distributed were expressed as mean $\pm \mathrm{SD}$, otherwise median and range (min, max) were used. For categorical variables, frequency and percentile were used to describe the data. Patients were categorized into two groups based on their answer to CGIC after 4 weeks treatment: "CGIC $=0$ " group vs "CGIC $\geq 1$ ".

Clinical characteristics between the two groups were compared using Chi-square or Mann-Whitney test. The comparison of IELT and NITBE before and after treatment was performed using paired $\mathrm{T}$ test. All statistical tests were two-tailed and a $P$ value of less than 0.05 was considered as statistically significant. The receiver-operating curve for treatment effect was also analyzed.

\section{Results}

One hundred seventy-two patients with PE received dapoxetine therapy (30 $\mathrm{mg}$ on-demand) for 4 weeks were included in this study. One hundred fifty-four patients were followed up and their data were analyzed and compared at baseline and after dapoxetine treatment. The mean age was $32.5 \pm 6.8$ (range: $21-61$ ) years. After 4 weeks treatment, there was an obvious improvement compared with the baseline regarding mean eIELT $(2.4 \pm 1.6$ $\min$ vs $1.0 \pm 0.7 \mathrm{~min}, P<0.001)$ and mean NITBE $(85.9 \pm$ 61.9 times vs $37.4 \pm 28.6$ times, $\mathrm{P}<0.001$ ) (Fig. 1 ).

Substantial improvements were found in all PEP measures (Fig. 2). At baseline, 9.7 and $0 \%$ of patients reported fair or good control over ejaculation which increased to 31.2 and $38.3 \%$ at week 4 , respectively $(P<0.001)$. At 


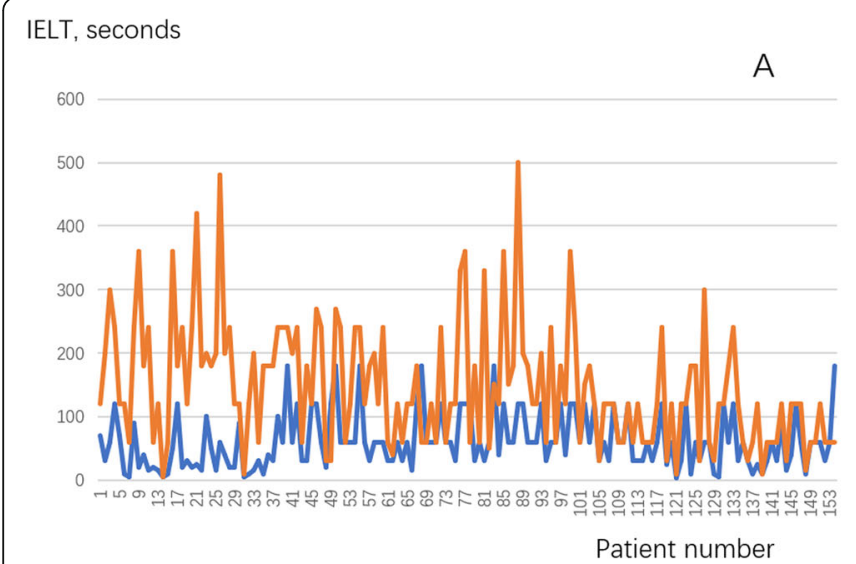

IELT(s) before treatment ___ IELT after treatment(s)
Number of thrust

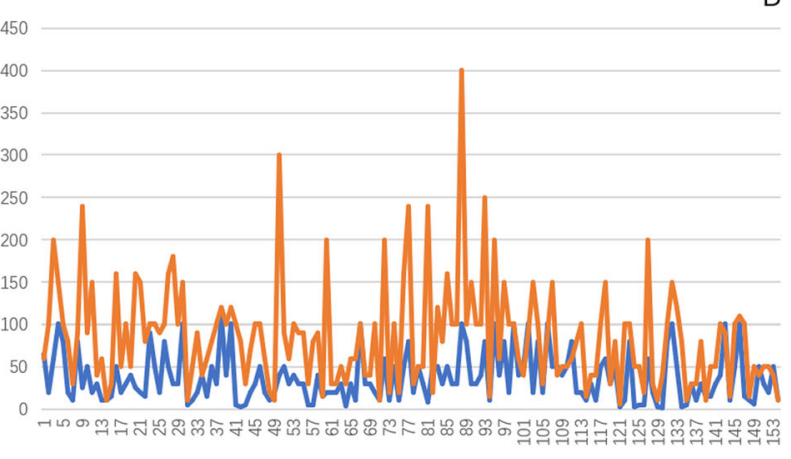

Patient number

Fig. 1 IELT distribution (a) and thrust times distribution (b). IELT: intravaginal ejaculation latency time

baseline, 20.1 and $0 \%$ of patients reported fair or good satisfaction with sexual intercourse which increased to 35.1 and $35.7 \%$ at week 4 , respectively $(P<0.001)$. At baseline, 30.5 and $41.6 \%$ of patients reported moderate or quite a bit of ejaculation-related personal distress which decreased to 19.5 and $7.8 \%$ at week 4 , respectively $(P<0.001)$. At baseline, 35.7 and $15.6 \%$ of patients reported moderate or quite a bit of interpersonal difficulty which decreased to 16.9 and $4.6 \%$ at week 4 , respectively $(P<0.001)$.

Overall, 108 patients (70.1\%) answered the CGIC question with "slightly better", "better" or "much better", and were categorized into "CGIC $\geq 1$ " group (Table 1). Regarding the clinical characteristics between the two groups, the mean NITBE at baseline was $28.0 \pm 22.0$ and $41.5 \pm 30.1$ times $(P=0.010)$ and mean PE severity at baseline measured by the PEDT was $15.7 \pm 2.7$ and $14.5 \pm 2.6$, respectively $(P=0.009)$, which indicated that the severity of $\mathrm{PE}$ and reduced NITBE prior to treatment may be associated with reduced treatment effect. A ROC curve was made to illustrate the relationship between PEDT scores and treatment effect (Fig. 3). Based on Youden index, the best cutoff value was 14.5 , with sensitivity $65.2 \%$ and specificity $57.4 \%$. A PEDT score of lower than 14.5 points would indicate a possibly better treatment effect. Notably, no significant difference in terms of baseline eIELT was found between the two groups $(56.3 \pm 44.4$ vs $63.0 \pm 42.5, p=$ 0.262 ). Baseline characteristics were also similar, including age, $\mathrm{PE}$ duration, previous treatment, and intercourse frequency (all $p>0.05$ ).

Regarding adverse effects, 21 patients (13.6\%) eventually discontinued dapoxetine treatment, and the reasons included lack of efficacy $(n=13)$, side effects $(n=5)$, and low frequency of sexual intercourse $(n=3)$. Of the patients who completed the 4-week treatment regimen, $72.2 \%$

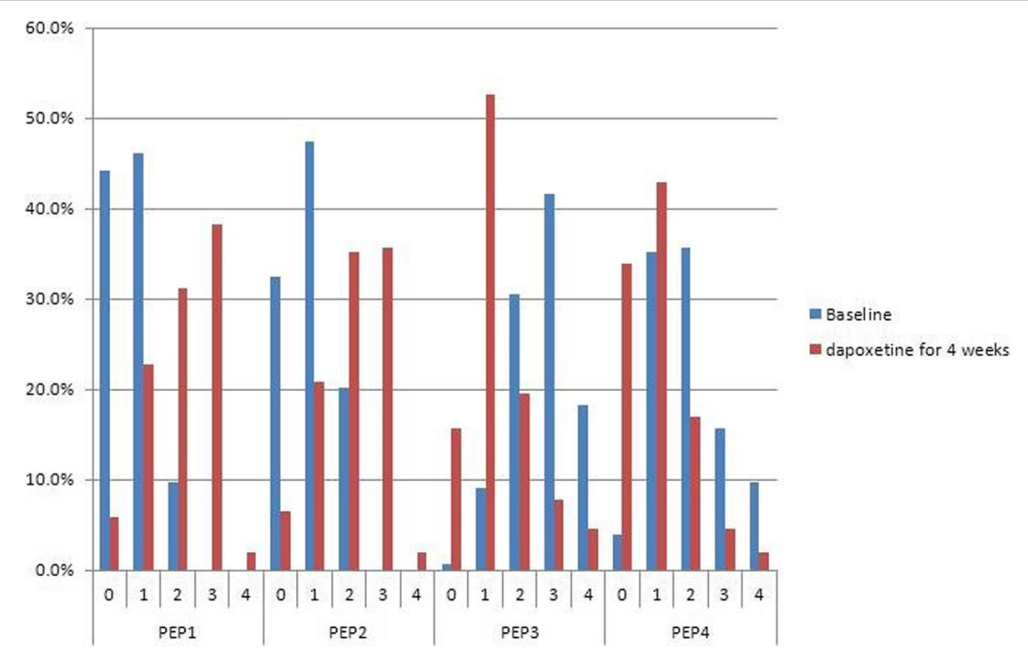

Fig. 2 Comparison of PEP before and after dapoxetine treatment. All PEP measures showed significant improvements with dapoxetine treatment at week 4 vs. baseline. PEP: premature ejaculation profile 
Table 1 Possible factors affecting the efficacy of dapoxetine $30 \mathrm{mg}$ treatment for 4 weeks

\begin{tabular}{|c|c|c|c|}
\hline \multirow[t]{2}{*}{ Parameters } & \multicolumn{2}{|l|}{ CGIC-C } & \multirow[t]{2}{*}{$P$} \\
\hline & $0(n=46)$ & $\geq 1(n=108)$ & \\
\hline Age (yeas, mean $\pm S D$ ) & $33.8 \pm 8.1$ & $31.9 \pm 6.1$ & 0.213 \\
\hline Marital Status: Married & $88.6 \%$ & $84.5 \%$ & 0.508 \\
\hline PE duration (years, mean $\pm S D$ ) & $6.4 \pm 5.7$ & $8.2 \pm 6.8$ & 0.058 \\
\hline Type of PE (\% of primary PE) & $84.8 \%$ & $70.4 \%$ & 0.060 \\
\hline Percentage of Intercourse frequency $\geq 2$ times & $43.4 \%$ & $53.7 \%$ & 0.245 \\
\hline Percentage of CP & $41.3 \%$ & $41.7 \%$ & 0.967 \\
\hline Percentage of previous SSRI use & $10.9 \%$ & $18.5 \%$ & 0.239 \\
\hline Percentage of previous Effective SSRI use & $10.0 \%$ & $28.6 \%$ & 0.097 \\
\hline Percentage of history of Circumcision or penile dorsal nerve transection & $28.3 \%$ & $34.3 \%$ & 0.467 \\
\hline PHQ-9(median [min, max]) & $6.0[0,27]$ & $6.0[0,26]$ & 0.835 \\
\hline GAD-7(median [min, max]) & $4.0[0,25]$ & $3.5[0,21]$ & 0.253 \\
\hline IELT (Seconds, mean \pm SD) & $56.3 \pm 44.4$ & $63.0 \pm 42.5$ & 0.262 \\
\hline NITBE (times, mean \pm SD) & $28.0 \pm 22.0$ & $41.5 \pm 30.1$ & 0.010 \\
\hline $\mathrm{PEDT}($ mean $\pm \mathrm{SD})$ & $15.7 \pm 2.7$ & $14.5 \pm 2.6$ & 0.009 \\
\hline
\end{tabular}

CGIC Clinical Global Impression of Change, CP chronic prostatitis, ED erectile dysfunction, GAD-7 General Anxiety Disorder Questionnaire, IELT intravaginal ejaculatory latency time, NITBE number of intravaginal thrusts before ejaculation, $P E$ premature ejaculation, $P E D T$ Premature Ejaculation Diagnostic Tool, $P H Q-9$ Patient Health Questionnaire-9 items, SD standard deviation, SSRI selective serotonin reuptake inhibitors

* statistical significance

would like to continue dapoxetine therapy. Treatment emergent adverse events (TEAEs) were reported in 32 patients $(20.8 \%)$, that mainly included headache (7.1\%), dizziness $(7.1 \%)$, nausea $(4.5 \%)$, somnolence $(1.9 \%)$ and nasal congestion (1.9\%). Most TEAEs were mild or moderate in severity and resolved without treatment.

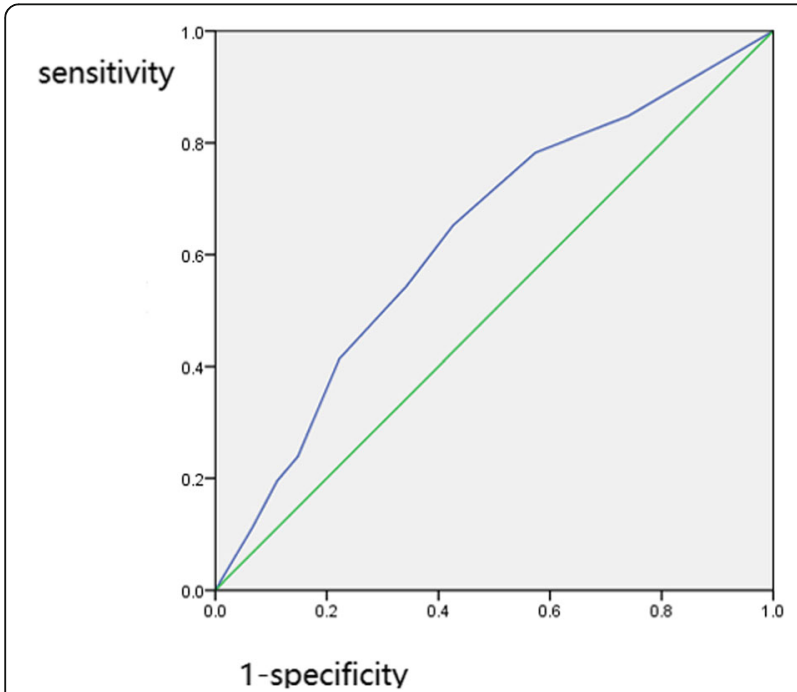

Fig. 3 The receiver-operating curve (ROC) of PEDT for treatment effect. Note: since higher PEDT score was related to poor treatment effect, the ROC curves are showing the prediction of $C G I C=0$, NOT CGIC $\geq 1$ (better results). PEDT: Premature Ejaculation Diagnostic Tool; CGIC: clinical global impression of change

\section{Discussion}

PE can interfere with sexual satisfaction, leading to decreased quality of life for the patients and their partners [16-18]. The mechanism for developing PE has not been fully elucidated, especially considering the role of physiological components. SSRIs had been introduced to the treatment of PE following the psychopharmacological studies regarding pathways that control ejaculation $[19,20]$. SSRIs could inhibit neuronal re-uptake of serotonin and subsequent potentiation of serotonin activity, and since the serotonergic neurotransmission is related to the pathways which control ejaculation, the delayed ejaculation is a commonly reported side effect $[21,22]$. However, these compounds are off-label, longacting and not ideal for on-demand use.

Based on a pooled analysis of five randomized, placebocontrolled, phase 3 clinical trials, dapoxetine, given ondemand, could delay ejaculation approximately $2.5-3$ fold and the therapy efficacy were better in those with lower baseline IELT. After 12 weeks $30 \mathrm{mg}$ or $60 \mathrm{mg}$ dapoxetine treatment, 62.1 and $71.7 \%$ of subjects reported that their PE was at least "slightly better", while in the control group this proportion was only $36.0 \%$ ( $P<0.001$ for both). Dapoxetine also improved overall sexual satisfaction, and reduced mental consequences including personal distress and interpersonal difficulty [4].

However, clinical data on dapoxetine treatment in real world practice is limited. Jiann et al. [23] reported the satisfaction rate and response rate were 45.0 and $74.6 \%$, respectively. We found dapoxetine treatment was wellaccepted by patients with PE and $70.1 \%$ of patients reportedly responded to dapoxetine treatment on demand. 
In this study, a total of $70.1 \%$ of patients reported that their PE was at least "slightly better" with dapoxetine treatment, and $40 \%$ of patients evaluated their treatment effect as "better". Yang et al. [24] reported similar results in Chinese men with PE. The rate of improvement and excellence with dapoxetine $30 \mathrm{mg}$ was 63.5 and 36.5\%, respectively. This reflects what was reported in five combined phase 3 trials of 6081 patients, whereby $62.1 \%$ of subjects taking dapoxetine $30 \mathrm{mg}$ reported that their PE was at least "slightly better" and 30.7\% reported "better" compared with $13.9 \%$ in the placebo arm $(P<0.001)$ [4]. Only those who reported that their PE was "better" were satisfied with sexual intercourse.

Dapoxetine was well-tolerated with $72.2 \%$ of patients stating that they would continue dapoxetine therapy. In our study, $64.4 \%$ of patients who discontinued dapoxetine treatment complained of lack of efficacy which was consistent with the main reasons for discontinuation of dapoxetine or SSRIs in phase 3 clinical trials [6, 25].

In the reported phase 3 trials, $64.9 \%$ of the study population were primarily categorized as patients with lifelong PE [4]. In our study, alongside primary PE incidence, we investigated other factors including, marital status, chronic prostatitis, intercourse frequency, prior use and reported efficacy of SSRIs, circumcision or penile dorsal nerve transection, ED and PE severity. While no correlation between efficacy of dapoxetine and the clinical factors listed above were identified, the severity of PE prior to treatment was found to be associated with the efficacy of dapoxetine.

Several factors may impact IELT and thus causing strong interpersonal differences which are reflected in the distribution of IELT values [26], including the performance of foreplay, the interval from last sexual experience, the gesture for sexual intercourse, the depth and force of thrusting, and the partner's vaginal lubrication [27]. We compared patient clinical characteristics and found that most factors would not affect CGI-C, including PE category, effective SSRI use, and estimated IELT. McMahon et al. [28] reported PE category $(P=0.5)$ and estimated baseline IELT $(P=0.16)$ did not affect CGIC rating of "slightly better" in 285 Asia-Pacific men with PE. Patients with a lower PEDT score and a higher NITBE at baseline responded better to dapoxetine.

Interestingly, eIELT was not significantly different between" CGIC $=0$ " and" CGIC $\geq 1$ " groups, suggesting that eIELT might be poorly estimated in the real-world setting. Lee et al. [7] compared stopwatch-determined IELT and eIELT in healthy men and found that eIELT was overestimated by approximately $1 \mathrm{~min}$.

The stopwatch-determined IELT measured by female partner might not be suitable in real world practice, although it is considered the most objective measure for PE evaluation in clinical trials. Therefore, there is a need for a simple, relative and reliable tool for PE evaluation in real world practice. In this study, we have introduced NITBE as a measure for PE evaluation. We found that baseline NITBE could predict dapoxetine treatment efficacy, and therefore, might be a useful measure for PE evaluation and might be more accurate than eIELT.

Waldinger et al. [29] proposed NITBE in 1994 and found that NITBE between patient assessment and partner assessment was consistent. However, NITBE was not used in subsequent studies. As a very convenient method, it would be possible to evaluate the IELT in daily life and might be a useful tool for PE diagnosis and treatment evaluation in real word practice; however, further validation is required.

Our study has several strengths, including the analysis of co-existing clinical factors that potentially impact PE, and we firstly used NITBE to evaluate PE and found better role of NITBE on PE evaluation than eIELT. In further studies, we will compare NITBE with stopwatch-determined IELT in healthy men and patients with PE. Despite its novel findings, the present study does have some limitations. This was a retrospective, open-label study and the sample size was small relative to clinical trials; there might be some inevitable bias due to the retrospective nature. A further prospective study would be required to reduce bias and increase the strength. A further limitation of the study was that objective stopwatch-determined IELT is difficult to obtain accurately in real world practice, thus clinical trials are required to testify the impact of frequency of penile movement on NITBE. Therefore, patients were included in the study based on the PEDT score, and patient-reported measures included PEP, CGIC and NITBE. Besides although PEP questionnaire has been used in previous studies focusing on Chinese patients [30], the linguistic validation has not been performed.

\section{Conclusions}

Dapoxetine treatment increased CGIC, eIELT and NITBE and was well-tolerated with an acceptable safety profile. Patients with less severe PE based on PEDT and higher NITBE seemed to have better efficacy with dapoxetine.

\section{Supplementary information}

Supplementary information accompanies this paper at https://doi.org/10. 1186/s12894-020-0580-3.

Additional file 1. Part of the database. Some information of the database, including IELT, NITBE and PEDT.

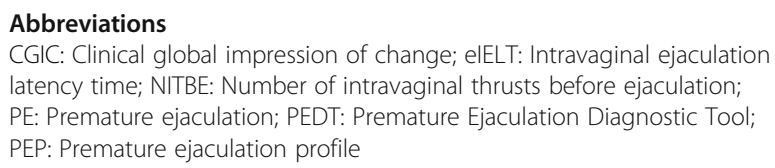

Acknowledgements

We thank Sainan Zhu for the help of critical analysis of data. 


\section{Authors' contributions}

JP, HongjunL, ZZ: Protocol/project development; JP, DF, HuixiL, YT, YY, WC, BG: Data collection or management; DF, HuixiL: Data analysis; JP, DF, HuixiL: Manuscript writing/editing; HongjunL, ZZ: Critical revision of the manuscript. All authors read and approved the final manuscript.

\section{Funding}

This study was funded by Beijing Natural Science Foundation (L182004) and the Fund for Fostering Young Scholars of Peking University Health Science Center (BMU2017PY009). The funding mainly helped in data collection.

\section{Availability of data and materials}

Parts of the data are attached in supplementary file. The datasets used and/ or analysed during the current study are available from the first author (Peng J \& Fang D) and corresponding author (Zhang Z) on reasonable request.

\section{Ethics approval and consent to participate}

The study received the ethics approval by committee of Peking University First Hospital. All procedures performed in studies involving human participants were in accordance with the ethical standards of the institutional and national research committee and with the 1964 Helsinki declaration and its later amendments or comparable ethical standards. Al patients agreed and signed the informed consent, that their information (including clinical information and surveillance) would be collected for scientific study and by published in professional medical journals.

\section{Consent for publication}

Not applicable.

\section{Competing interests}

The authors declare that they have no competing interests.

Received: 31 March 2019 Accepted: 23 January 2020

Published online: 03 February 2020

\section{References}

1. Porst H, Montorsi F, Rosen RC, Gaynor L, Grupe S, Alexander J. The premature ejaculation prevalence and attitudes (PEPA) survey: prevalence, comorbidities, and professional help-seeking. Eur Urol. 2007;51(3):816-23.

2. Laumann EO, Paik A, Rosen RC. Sexual dysfunction in the United States: prevalence and predictors. JAMA. 1999;281(6):537-44.

3. Modi NB, Dresser MJ, Simon M, Lin D, Desai D, Gupta S. Single- and multipledose pharmacokinetics of dapoxetine hydrochloride, a novel agent for the treatment of premature ejaculation. J Clin Pharmacol. 2006;46(3):301-9.

4. McMahon CG, Althof SE, Kaufman JM, Buvat J, Levine SB, Aquilina JW, Tesfaye F, Rothman M, Rivas DA, Porst H. Efficacy and safety of dapoxetine for the treatment of premature ejaculation: integrated analysis of results from five phase 3 trials. J Sex Med. 2011;8(2):524-39.

5. Russo A, Capogrosso P, Ventimiglia E, La Croce G, Boeri L, Montorsi F, Salonia A. Efficacy and safety of dapoxetine in treatment of premature ejaculation: an evidence-based review. Int J Clin Pract. 2016;70(9):723-33.

6. Mondaini N, Fusco F, Cai T, Benemei S, Mirone V, Bartoletti R. Dapoxetine treatment in patients with lifelong premature ejaculation: the reasons of a "Waterloo". Urology. 2013;82(3):620-4.

7. Lee WK, Cho ST, Lee YS, Lee YG, Oh CY, Yoo C, Cho JS, Shin TY, Lee SK, Lee SH, Ko K, Yang DY. Can estimated intravaginal ejaculatory latency time be used interchangeably with stopwatch-measured intravaginal ejaculatory latency time for the diagnosis of lifelong premature ejaculation? Urology. 2015;85(2):375-80.

8. Kam SC, Han DH, Lee SW. The diagnostic value of the premature ejaculation diagnostic tool and its association with intravaginal ejaculatory latency time. J Sex Med. 2011;8(3):865-71.

9. Huang $Y P$, Chen B, Ping P, Wang HX, Hu K, Zhang T, Yang H, Jin $Y$, Yang $Q$, Huang YR. The premature ejaculation diagnostic tool (PEDT): linguistic validity of the Chinese version. J Sex Med. 2014;11(9):2232-8.

10. Jiang H, Liu D, Deng C, Shang X, Hong K, Deng J, Wang Z, Yang H, Shi Y, Huang Y, Wang P, Zhang Y, Liang J, Shen B, Li F, Zhang C. Reliability and validity of the Chinese version of the premature ejaculation diagnostic tool. Natl J Androl. 2015;21(7):598-603.
11. Patrick DL, Giuliano F, Ho KF, Gagnon DD, McNulty P, Rothman M. The premature ejaculation profile: validation of self-reported outcome measures for research and practice. BJU Int. 2009;103(3):358-64.

12. Kroenke K, Spitzer RL, Williams JB. The PHQ-9: validity of a brief depression severity measure. J Gen Intern Med. 2001;16(9):606-13.

13. Ruiz MA, Zamorano E, García-Campayo J, Pardo A, Freire O, Rejas J. Validity of the GAD-7 scale as an outcome measure of disability in patients with generalized anxiety disorders in primary care. J Affect Disord. 2011;128(3):277-86.

14. Wang W, Bian Q, Zhao Y, Li X, Wang W, Du J, Zhang G, Zhou Q, Zhao M. Reliability and validity of the Chinese version of the patient health questionnaire (PHQ-9) in the general population. Gen Hosp Psychiatry. 2014; 36(5):539-44.

15. Zeng QZ, He YL, Liu H, Miao JM, Chen JX, Xu HN, Wang JY. Reliability and validity of Chinese version of the Generalized Anxiety Disorder 7-item (GAD7) scale in screening anxiety disorders in outpatients from traditional Chinese internal department. Chin Mental Health J. 2013;27(3):163-8 [Article in Chinese].

16. Symonds T, Roblin D, Hart K, Althof S. How does premature ejaculation impact a man s life? J Sex Marital Ther. 2003;29(5):361-70.

17. Montague DK, Jarow J, Broderick GA, Dmochowski RR, Heaton JP, Lue TF, Nehra A, Sharlip ID. AUA erectile dysfunction guideline update panel. AUA guideline on the pharmacologic management of premature ejaculation. J Urol. 2004;172(1):290-4.

18. Byers ES, Grenier G. Premature or rapid ejaculation: heterosexual couples' perceptions of men's ejaculatory behavior. Arch Sex Behav. 2003;32(3):261-70.

19. Waldinger MD, Berendsen HH, Blok BF, Olivier B, Holstege G. Premature ejaculation and serotonergic antidepressants-induced delayed ejaculation: the involvement of the serotonergic system. Behav Brain Res. 1998;92(2):111-8.

20. Waldinger MD. The neurobiological approach to premature ejaculation. J Urol. 2002;168(6):2359-67.

21. McMahon CG, Touma K. Treatment of premature ejaculation with paroxetine hydrochloride as needed: 2 single-blind placebo controlled crossover studies. J Urol. 1999;161(6):1826-30.

22. Waldinger MD, Hengeveld MW, Zwinderman AH, Olivier B. Effect of SSRI antidepressants on ejaculation: a double-blind, randomized, placebocontrolled study with fluoxetine, fluvoxamine, paroxetine, and sertraline. J Clin Psychopharmacol. 1998;18(4):274-81.

23. Jiann $B P$, Huang $Y J$. Assessing satisfaction in men with premature ejaculation after dapoxetine treatment in real-world practice. Int J Clin Pract. 2015;69(11):1326-33

24. Yang L, Luo L, Chen XF, Fan JH, Liu RM, Wang XN, Nan XY, Zhang Y, Lin XF, Wang MZ, Xing JP, Yang ZS, Jian BL, He H, Wu DP, He DL. Efficacy and tolerability of dapoxetine in the treatment of premature ejaculation. Zhonghua Nan Ke Xue. 2015;21(10):892-5 [Article in Chinese].

25. Salonia A, Rocchini L, Sacca' A, Pellucchi F, Ferrari M, Carro UD, Ribotto P, Gallina A, Zanni G, Deho' F, Rigatti P, Montorsi F. Acceptance of and discontinuation rate from paroxetine treatment in patients with lifelong premature ejaculation. J Sex Med. 2009;6(10):2868-77.

26. Waldinger MD, Zwinderman AH, Olivier B, Schweitzer DH. Geometric mean IELT and premature ejaculation: appropriate statistics to avoid overestimation of treatment efficacy. J Sex Med. 2008;5(2):492-9.

27. McMahon CG, Althof SE, Waldinger MD, Porst H, Dean J, Sharlip ID, Adaikan PG, Becher E, Broderick GA, Buvat J, Dabees K, Giraldi A, Giuliano F, Hellstrom WJ, Incrocci L, Laan E, Meuleman E, Perelman MA, Rosen RC, Rowland DL, Segraves R. An evidence-based definition of lifelong premature ejaculation: report of the International Society for Sexual Medicine (ISSM) ad hoc committee for the definition of premature ejaculation. J Sex Med. 2008;5(7):1590-606.

28. McMahon C, Lee SW, Kim SW, Moon du G, Kongkanand A, Tantiwongse K. The Asia-Pacific flexible dose study of dapoxetine and patient satisfaction in premature ejaculation therapy: the PASSION study. Sex Med. 2016;4(1):e18-27.

29. Waldinger MD, Hengeveld MW, Zwinderman AH. Paroxetine treatment of premature ejaculation: a double-blind, randomized, placebo-controlled study. Am J Psychiatry. 1994;151(9):1377-9.

30. Ya Z, Zhang X, Shen L, Peng D, Tang D, Huang Z, Cheng P, Dou X, Mao J, Gao J, Liang C. Efficacy and discontinuation reasons of dapoxetine in the treatment of lifelong premature ejaculation. Chin J Androl. 2018;32(3):12-6.

\section{Publisher's Note}

Springer Nature remains neutral with regard to jurisdictional claims in published maps and institutional affiliations. 